\section{Axial length and intraoperative posterior vitreous detachment as predictive factors for surgical outcomes of diabetic vitrectomy}

WK Song', SS Kim², J-H Yi', SH Byeon', HJ Koh', SC Lee ${ }^{1}$ and OW Kwon'
${ }^{1}$ Department of Ophthalmology, Institute of Vision Research, Yonsei University College of Medicine, Seoul, Republic of Korea

\section{Introduction}

According to the Early Treatment Diabetic Retinopathy Study, at least $5 \%$ of eyes receiving optimal medical treatment have progressive retinopathy that requires laser treatment and pars plana vitrectomy (PPV). Advances in instrumentation and surgical techniques have improved the outcomes of diabetic vitrectomy. However, the functional results are occasionally disappointing, even in eyes with successful operations, and vitreous surgery has a potential for severe complications in diabetic eyes. ${ }^{1}$

General categories of surgical indications for diabetic vitrectomy include eyes with media opacity and vitreo-retinal traction. ${ }^{1}$ Tractional retinal detachment (TRD) with progressive fibrovascular proliferation (FVP) is an important indication for vitrectomy. If the posterior hyaloid is attached and has a broad vitreoretinal adhesion (VRA) to the underlying retina, extensive dissection of the FVP may lead to more intraoperative complications. ${ }^{2}$ The lack of posterior vitreous detachment (PVD) is presumed to be an important factor associated with visual prognosis in these patients, ${ }^{3}$ but it has not been previously evaluated.
${ }^{2}$ Department of University College of Medicine, Gangnam Severance Hospital, Seoul, Republic of Korea

Correspondence: SS Kim, Department of Ophthalmology, Yonsei University College of Medicine, Gangnam Severance Hospital, 712 Eonjuro, Gangnam-Gu, Seoul, Republic of Korea Tel: + 8222019 3443; Fax: + 82234631049 E-mail: semekim@ naver.com

Received: 12 March 2009 Accepted in revised form: 1 December 2009 Published online: 15 January 2010

The abstract of this article was presented as a poster at the 2007 annual meeting of the Association for Research in Vision and Ophthalmology Ophthalmology, Yonsei 
In diabetic eyes with media opacity, preoperative ultrasound (US) examination may reveal the presence of PVD, but the detailed vitreo-macular configuration cannot be accurately evaluated. Occasionally, surgeons may encounter strong VRA on the macula during vitrectomy in cases with PVD detected by US. Accurate and adequate diagnostic tools for evaluating the vitreous is not yet available, and therefore PVD is perhaps the least accurate diagnosis entertained by ophthalmologists on a daily basis. ${ }^{4}$

On the other hand, axial length is easily measured with preoperative US biometry, regardless of media opacity. In the normal population, PVD is reported more frequently in moderate or high myopia than in emmetropia, and axial length is a significant factor in the development of PVD in high myopia. ${ }^{5-7}$

To estimate surgical outcomes of diabetic vitrectomy in relation to the severity of VRA, we studied the relationship between the intraoperatively confirmed PVD status and the preoperatively measured axial length in patients undergoing diabetic vitrectomy, and examined their influence on surgical outcomes.

\section{Materials and methods}

This study was a retrospective, consecutive, non-randomized, interventional case series. It was conducted in accordance with the principles of the Declaration of Helsinki and performed under the approval of the Institutional Review Board of Gangnam Severance Hospital, Yonsei University College of Medicine, Seoul, Republic of Korea. We reviewed the clinical records for diabetic retinopathy patients who had undergone diabetic vitrectomy and were evaluated at least 6 months postoperatively by a single surgeon (SSK) between March 2002 and December 2005 at Gangnam Severance Hospital. When both eyes met the inclusion criteria, one randomly selected eye was included. Patients included in this study had no previous PPV or intravitreal injections aside from laser photocoagulation or posterior subtenon steroid injections for diabetic macular oedema and/or proliferative diabetic retinopathy (PDR). Cases with diabetic macular oedema were excluded because the nature and prognosis may differ from PDR cases. Eyes with a history of ocular trauma, intraocular surgery other than cataract surgery, and uveitis were also excluded.

The following preoperative data were obtained for each patient: age, gender, best corrected Snellen visual acuity (BCVA), axial length measured with ultrasound biometry, combined systemic diseases, preoperative scattered laser treatment (PRP), and preoperative posterior subtenon steroid injection. BCVA was converted to units of the logarithm of the minimum angle of resolution (logMAR BCVA) to facilitate statistical comparison. ${ }^{8}$ All patients underwent standard 20-G 3-port PPV on their affected eyes. PVD status was classified into three groups by intraoperative evaluation of the configuration of VRA around the macula and optic nerve. Broad VRA without separation of the posterior hyaloid from the macula and optic nerve was classified as no PVD, PVD in the macular area with attachment at the disc was classified as incomplete PVD, and complete detachment was classified as complete PVD $(0,1$, and 2, respectively, were entered in statistical analysis).

Phacoemulsification and posterior chamber intraocular lens (PCIOL) implantation were performed for eyes with significant cataracts. Air-fluid exchange and silicone oil tamonade (SOT) were performed when multiple iatrogenic retinal breaks developed in more than two quadrants. All patients underwent posterior subtenon triamcinolone acetonide injection at the end of the surgery, followed by a standard regimen of antibiotics. Anatomical success was defined as an attached retina with vision better than light perception and an intraocular pressure in the normal range $(5-21 \mathrm{~mm} \mathrm{Hg})$. Functional success was defined as a BCVA at the final visit better than 20/100 ( $\log$ MAR 0.7). Car-driving capable visual acuity was defined as better than 20/40 (logMAR 0.3). Statistical analyses were performed using SAS/STAT Software (version 8.2, SAS America, Cary, NC, USA). A $P$-value less than 0.05 was considered statistically significant.

\section{Results}

One hundred and fifteen eyes of 50 male and 65 female patients were included in this study. Intraoperatively, we classified 44 eyes with no PVD, 23 eyes with incomplete PVD, and 48 eyes with complete PVD. Complete PVD patients had significantly longer axial length than the no PVD group and incomplete PVD group (ANOVA between the three groups; $P=0.0001$, multiple comparisons by Bonferroni method; $P<0.0001$ and $P=0.007$ each) (Table 1). Age and preoperative logMAR BCVA showed significant differences in the three groups (ANOVA; $P=0.02$ and $P=0.001$, respectively). In multiple comparisons by the Bonferroni method, age in the complete PVD group was higher than in the no PVD group, showing that complete PVD is more common in the aged diabetic vitrectomy patients $(P=0.03)$. The group with incomplete PVD showed better preoperative logMAR BCVA compared with the no PVD group and complete PVD group (multiple comparisons by Bonferroni method; $P=0.001, P=0.005$, respectively). The poorer preoperative BCVA in these groups were because of the high incidence of macula off TRD (97.7\%) in the no PVD group and vitreous haemorrhage $(68.7 \%)$ in the complete PVD group. Preoperative scatter 
Table 1 Baseline characteristics

\begin{tabular}{|c|c|c|c|c|c|}
\hline Charateristics & $\begin{array}{l}\text { Overall } \\
(n=115)\end{array}$ & $\begin{array}{l}\text { No } P V D \\
(n=44)\end{array}$ & $\begin{array}{l}\text { Incomplete PVD } \\
\quad(n=23)\end{array}$ & $\begin{array}{l}\text { Complete PVD } \\
\quad(n=48)\end{array}$ & P-value \\
\hline \multicolumn{6}{|l|}{ Age (years) } \\
\hline Mean & 54.5 & 50.7 & 56.7 & 56.8 & ${ }^{\mathrm{a}} 0.02$ \\
\hline \multicolumn{6}{|l|}{ Gender (\%) } \\
\hline Male & $50(43.5)$ & $14(31.8)$ & $10(43.5)$ & $26(54.2)$ & ${ }^{\mathrm{b}} 0.09$ \\
\hline Female & $65(56.5)$ & $30(68.2)$ & $13(56.5)$ & $22(45.8)$ & \\
\hline \multicolumn{6}{|l|}{ Preoperative BCVA } \\
\hline Mean $\log$ MAR (mean Snellen equivalent) & $1.70(20 / 1002)$ & $1.84(20 / 1262)$ & $1.29(20 / 399)$ & $1.77(10 / 1262)$ & ${ }^{\mathrm{a}} 0.001$ \\
\hline \multicolumn{6}{|l|}{ Axial length (mm) } \\
\hline \multirow[t]{3}{*}{ Mean } & 22.93 & 22.56 & 22.64 & 23.4 & a 0.0001 \\
\hline & \multirow{2}{*}{\multicolumn{5}{|c|}{$\begin{array}{l}\text { No PVD vs complete PVD: }{ }^{*} P<0.0001 \\
\text { Incomplete PVD vs complete PVD: }{ }^{*} P=0.007\end{array}$}} \\
\hline & & & & & \\
\hline Pseudophakia before surgery $(\%)$ & $15(14.0)$ & $4(9.1)$ & $2(8.7)$ & $9(18.8)$ & b 0.3 \\
\hline Scatter laser treatment before surgery (\%) & $74(64.3)$ & $24(54.5)$ & $17(73.9)$ & $33(68.8)$ & ${ }^{b} 0.2$ \\
\hline
\end{tabular}

BCVA, best corrected visual acuity; logMAR, logarithm of the minimum angle of resolution; PVD, posterior vitreous detachment.

${ }^{*} P$-value in post hoc test with Bonferroni method.

$P$-value of a ANOVA and ${ }^{b}$ Pearson $\chi^{2}$ test.

laser photocoagulation and lens status showed no significant difference between the groups with different PVD statuses $\left(\chi^{2} ; P=0.2, P=0.3\right)$. Thirty-three patients received vitrectomy because of vitreous haemorrhage, 37 because of TRD threatening the macula, 43 because of TRD involving the macula, and two because of neovascular glaucoma with vitreous haemorrhage (Table 2). The distribution of diagnoses showed differences in the three PVD groups $\left(\chi^{2} ; P<0.0001\right)$. A majority of the cases with broad VRA had TRD involving the macular area (97.7\%). All eyes with incomplete PVD showed TRD, but spared the macular area. Eyes with complete PVD had a low incidence of TRD (29.2\%), and most cases had only vitreous haemorrhage with few FVPs $(68.7 \%)$. Vitrectomy combined with phacoemulsification and PCIOL implantation was performed in $92 \%$ of the 100 phakic eyes and the rates of concomitant phacoemulsification were not different among the groups $\left(\chi^{2} ; P=0.1\right)$. The extent of VRA was closely associated with a higher rate of SOT $\left(\chi^{2} ; P<0.0001\right)$. Cases with less PVD had a higher postoperative rate of recurrence of the tractional epiretinal membrane and TRD $\left(\chi^{2} ; P=0.01, P=0.04\right)$. The rate of neovascular glaucoma, cataract progression, postoperative vitreous haemorrhage, and rhegmatogenous retinal detachment were not different among the groups. Univariate logistic regression analysis showed that an increase in axial length was a significant predictor of anatomical success (OR 2.361, 1.088-5.123 95\% CI). Chi-square analysis showed that the PVD status was associated with improvement of visual acuity after vitrectomy $(P=0.01)$, attaining a final $B C V A>20 / 200$
$(P=0.002)$, a final BCVA $>20 / 100(P<0.0001)$, and final $\mathrm{BCVA}>20 / 40(P=0.008)$.

Multiple logistic regression analysis with the stepwise method was performed for anatomical and functional outcomes. Age, gender, preoperative logMAR BCVA, PVD status, axial length, preoperative scatter laser, preoperative subtenon steroid injection, operative diagnosis, pseudophakia before surgery, surgery combined with phacoemulsification, silicone oil tamponade, and gas tamponade were included as independent variables and values of variance inflation factors showed no significant collinearity between these variables. It showed that the PVD status is a significant prognostic factor for attaining a BCVA $>20 / 100$ (OR $1.932,1.143-3.266,95 \% \mathrm{CI})$ and $\mathrm{BCVA}>20 / 40$ at the final visit (OR 1.829, 1.067-3.133, 95\% CI) (Table 3). Postoperative complications such as NVG, recurrence of tractional ERM, and TRD showed negative correlation with anatomical and functional success (Spearman's rank correlation) (Table 4).

\section{Discussion}

It has been previously suggested that vitreo-retinal attachments in PDR could possibly serve as a predictive factor of visual and anatomic outcome following diabetic vitrectomy. ${ }^{2}$ Eliott classified diabetic vitrectomy cases into six groups according to intraoperative PVD status. ${ }^{3}$ He presumed that the extent of VRA could be useful in a grading system for eyes undergoing diabetic vitrectomy. Ikeda et $a l^{9}$ reported these relationships in a subset of 26 eyes with asteroid hyalosis accompanying PDR treated 
Table 2 Operative findings and outcomes

\begin{tabular}{|c|c|c|c|c|c|}
\hline Charateristics & $\begin{array}{c}\text { Overall } \\
(n=115)\end{array}$ & $\begin{array}{l}\text { No PVD } \\
(n=44)\end{array}$ & $\begin{array}{c}\text { Incomplete PVD } \\
\quad(n=23)\end{array}$ & $\begin{array}{c}\text { Complete PVD } \\
\quad(n=48)\end{array}$ & P-value \\
\hline Operative diagnosis (\%) & & & & & ${ }^{\mathrm{a}}<0.0001$ \\
\hline VHNERM & $33(28.7)$ & 0 & 0 & $33(68.7)$ & \\
\hline MATRD & $37(32.2)$ & 0 & $23(100)$ & $14(29.2)$ & \\
\hline MDTRD & $43(37.4)$ & $43(97.7)$ & 0 & 0 & \\
\hline VHNVG & $2(1.7)$ & $1(2.3)$ & 0 & $1(2.1)$ & \\
\hline Surgery combined with phacoemulsification (\%) & $92(80)$ & $39(88.6)$ & $20(87)$ & $33(68.8)$ & ${ }^{\mathrm{a}} 0.1$ \\
\hline Silicone oil tamponade (\%) & $43(37.4)$ & $32(72.7)$ & $4(17.4)$ & 7 (14.6) & ${ }^{\mathrm{a}}<0.0001$ \\
\hline \multicolumn{6}{|l|}{ Postoperative complication } \\
\hline Neovascular glaucoma (\%) & $8(7)$ & $1(2.3)$ & $3(13)$ & $4(8.3)$ & ${ }^{\mathrm{a}} 0.2$ \\
\hline Membrane reproliferation & & & & & \\
\hline Epiretinal membrane (\%) & $35(30.4)$ & $16(36.4)$ & $7(30.4)$ & 2(4.2) & ${ }^{\mathrm{a}} 0.01$ \\
\hline Tractional RD (\%) & $6(5.2)$ & $5(11.4)$ & $1(4.3)$ & $0(0)$ & ${ }^{\mathrm{a}} 0.04$ \\
\hline Follow-up time (months) & 25.6 & 24.9 & 26.7 & 25.6 & ${ }^{\mathrm{b}} 0.5$ \\
\hline Anatomical success (\%) & $104(90.4)$ & $37(84.1)$ & $20(87)$ & $47(97.9)$ & a 0.06 \\
\hline \multicolumn{6}{|l|}{ Final $B C V A$} \\
\hline Mean logMAR (mean Snellen equivalent) & $0.95(20 / 200)$ & $1.35(20 / 450)$ & $0.73(20 / 100)$ & $0.68(20 / 100)$ & ${ }^{\mathrm{b}}<0.0001$ \\
\hline Improvement of BCVA (\%) & $89(77.4)$ & $28(63.6)$ & $18(78.3)$ & $43(89.6)$ & ${ }^{\mathrm{a}} 0.01$ \\
\hline Improvement of $0.2 \log$ MAR units (\%) & $84(73)$ & $28(63.6)$ & $14(60.9)$ & $42(87.5)$ & ${ }^{\mathrm{a}} 0.01$ \\
\hline $\mathrm{BCVA}>20 / 200$ & $75(65.2)$ & $20(45.5)$ & $17(73.9)$ & $38(79.2)$ & ${ }^{\mathrm{a}} 0.002$ \\
\hline BCVA $>20 / 100$ & $54(47)$ & 9 (20.5) & $16(69.6)$ & $29(60.4)$ & ${ }^{\mathrm{a}}<0.0001$ \\
\hline $\mathrm{BCVA}>20 / 40$ & $28(24.3)$ & $4(9.1)$ & 9 (39.1) & 15 (31.3) & ${ }^{\mathrm{a}} 0.008$ \\
\hline
\end{tabular}

BCVA, best corrected Snellen visual acuity; PVD, posterior vitreous detachment; logMAR, logarithm of the minimum angle of resolution; MATRD, macula attached tractional retinal detachment; MDTRD, macula detached tractional retinal detachment; VHNERM, vitreous hemorrhage without epiretinal membrane; VHNVG, vitreous hemorrhage with neovascular glaucoma.

$P$-value of ${ }^{\mathrm{a}}$ Pearson $\chi^{2}$ test and ${ }^{\mathrm{b}}$ ANOVA.

by PPV. Generally, in non-diabetic eyes, complete PVD occurred less often in eyes with asteroid hyalosis, and partial PVD occurred more often in eyes with asteroid hyalosis than in control eyes. In their study, most of the cases had no PVD and in $76.9 \%$ of the cases iatrogenic retinal breaks developed during artificial detachment, which is high compared with the rate in general diabetic vitrectomy. ${ }^{10}$ VRA is closely related to the presence of PVD, and enzymatic induction of PVD was reported as beneficial in surgical management of diabetic retinopathy. ${ }^{11}$

In this study, we found that PVD status (VRA at the macula) may be an important predictor of functional outcomes following diabetic vitrectomy. VRA in diabetic eyes is associated with fibrovascular complex formation. Eyes with extensive VRA required mechanical separation of the tight hyaloid membrane, which resulted in higher rates of membrane reproliferation $(36.4 \%)$ and postoperative TRD $(11.4 \%)$, contributing to poorer final visual outcomes. Membrane peeling causes more trauma to the macula, intraoperative haemorrhage, iatrogenic retinal tears, and consequent postoperative glial tissue proliferation. The presence of postoperative complications including neovascular glaucoma, epiretinal membrane formation, and TRD resulted in poorer outcomes. As such, the results appear similar to those for PDR diabetic vitrectomy in other reports. ${ }^{1,12}$

Recently, optical coherence tomography (OCT) has been used to obtain detailed views of the vitreo-macular configuration enabling evaluation of the extent of VRA preoperatively. However, OCT has limitations in eyes with media opacity. In such eyes, PVD is often diagnosed by ultrasonography. However, the presence of PVD can never be definitively established because the level of resolution is not sufficient to reliably image the posterior vitreous cortex. ${ }^{4}$ Surgeons may occasionally encounter unexpected, severe conditions because of extensive and broad VRAs. We hypothesized that a correlation between axial length and PVD status might be helpful in such circumstances. Ocular axial length is easily measured with ultrasound biometry and is a routine evaluation for lens surgery, which may be combined with PPV. A positive correlation between onset age of PVD and refractive error was reported in a study of myopia, emmetropia, and hyperopia. ${ }^{13}$ The axial length of the eye is an important factor responsible for the development of 
Table 3 Multiple logistic regression analysis results

\begin{tabular}{lllll}
\hline Postoperative outcomes & Factor & *P-value & Odd ratio & CI 95\% \\
\hline Anatomical success & PVD status & 0.05 & 2.265 & $0.980-5.238$ \\
& Scatter laser treatment before surgery & 0.09 & 3.090 & $0.821-11.628$ \\
Functional outcomes & & & \\
Improvement of BCVA & PVD status & 0.07 & 1.902 & $0.933-3.876$ \\
& Preoperative logMAR BCVA & 0.004 & 2.347 & $0.815-6.761$ \\
& Gender & 0.1 & 0.437 & $0.150-1.268$ \\
& Scatter laser treatment before surgery & 0.1 & 0.241 & $0.073-0.801$ \\
& Silicone oil tamponade & 0.02 & 3.627 & $1.479-8.895$ \\
Improvement of BCVA by $0.2 \log M A R$ & PVD status & 0.06 & 1.956 & $0.964-3.969$ \\
& Preoperative logMAR BCVA & 0.008 & 0.454 & $0.160-1.284$ \\
& Gender & 0.1 & 0.227 & $0.069-0.742$ \\
BCVA $>20 / 200$ & Silicone oil tamponade & 0.01 & 3.122 & $1.330-7.331$ \\
& Age & 0.1 & 2.085 & $0.875-4.970$ \\
& Operative Diagnosis & 0.06 & 0.968 & $0.929-1.008$ \\
& Scatter laser treatment before surgery & 0.09 & 0.547 & $0.288-1.036$ \\
BCVA $>20 / 100$ & Silicone oil tamponade & 0.04 & 0.356 & $0.130-0.971$ \\
& PVD status & 0.01 & 1.932 & $1.143-3.266$ \\
BCVA $>20 / 40$ & Preoperative logMAR BCVA & 0.02 & 0.457 & $0.172-1.213$ \\
& Silicone oil tamponade & 0.1 & 0.449 & $0.223-0.907$ \\
\hline BCVA & PVD status & 0.02 & 1.829 & $1.067-3.133$ \\
& Preoperative logMAR BCVA & 0.03 & 0.481 & $0.241-0.959$ \\
\hline
\end{tabular}

$\mathrm{BCVA}$, best corrected Snellen visual acuity; CI, confidence interval; logMAR, logarithm of the minimum angle of resolution; PVD, posterior vitreous detachment.

${ }^{*} P$-values in multiple logistic regression analysis stepwise method with age, gender, preoperative BCVA, PVD status, axial length, preoperative scatter laser, preoperative subtenon steroid injection, operative diagnosis, pseudophakia before surgery, surgery combined with phacoemulsification, silicone oil tamponade, gas tamponade as independent variables.

Table 4 Spearman's rank correlation analysis results

\begin{tabular}{|c|c|c|c|}
\hline Postoperative outcomes & Factor & P-value & Coefficient \\
\hline \multirow[t]{3}{*}{ Anatomical success } & Neovascular glaucoma & 0.000 & -0.376 \\
\hline & Tractional epiretinal membrane & 0.06 & -0.174 \\
\hline & Tractional retinal detachment & 0.000 & -0.588 \\
\hline \multicolumn{4}{|l|}{ Functional outcomes } \\
\hline \multirow[t]{3}{*}{ Improvement of BCVA } & Neovascular glaucoma & 0.000 & -0.342 \\
\hline & Tractional epiretinal membrane & 0.004 & -0.263 \\
\hline & Tractional retinal detachment & 0.000 & -0.341 \\
\hline \multirow[t]{3}{*}{ Improvement of BCVA by $0.2 \log \mathrm{MAR}$} & Neovascular glaucoma & 0.001 & -0.296 \\
\hline & Tractional epiretinal membrane & 0.001 & -0.310 \\
\hline & Tractional retinal detachment & 0.001 & -0.298 \\
\hline \multirow[t]{3}{*}{ BCVA $>20 / 200$} & Neovascular glaucoma & 0.01 & -0.231 \\
\hline & Tractional epiretinal membrane & 0.004 & -0.265 \\
\hline & Tractional retinal detachment & 0.01 & -0.239 \\
\hline \multirow[t]{3}{*}{ BCVA $>20 / 100$} & Neovascular glaucoma & 0.04 & -0.189 \\
\hline & Tractional epiretinal membrane & 0.01 & -0.231 \\
\hline & Tractional retinal detachment & 0.1 & -0.142 \\
\hline \multirow[t]{3}{*}{$\mathrm{BCVA}>20 / 40$} & Neovascular glaucoma & 0.09 & -0.155 \\
\hline & Tractional epiretinal membrane & 0.03 & -0.198 \\
\hline & Tractional retinal detachment & 0.6 & -0.042 \\
\hline
\end{tabular}

BCVA, best corrected Snellen visual acuity; logMAR, logarithm of the minimum angle of resolution. 
PVD in patients with high myopia. ${ }^{5-7}$ However, little was known about the relationship between axial length and diabetic retinopathy. Pierro et $a l^{14}$ reported that axial length is shorter in diabetic patients than in non-diabetic patients. Dogru et al ${ }^{15}$ reported that high myopia has a protective influence regarding the development of diabetic retinopathy.

This study showed (at univariate level) that longer axial length was associated with anatomical success of diabetic vitrectomy. Also, a shorter axial length reflected a higher chance of broad VRA. In cases with media opacity where evaluation of the vitreo-retinal relationship is impaired, surgeons should not ignore that eyes with short axial length may be associated with more extensive VRAs. When performing diabetic vitrectomy in PDR patients with a lack of PVD in whom extensive VRA is anticipated, both careful decision-making regarding the timing of the surgery and extensive informed consent are necessary. Further studies examining the relationship between preoperative treatments that induce artificial PVD, such as intravitreal injection procedures, and surgical outcomes are necessary.

\section{Summary}

What was known before

- The lack of posterior vitreous detachment is presumed to be an important factor associated with the visual prognosis in diabetic vitrectomy patients, but it has not previously been evaluated.

What this study adds

- This study demonstrated that axial length is highly correlated with intraoperatively assessed posterior vitreous detachment status in diabetic eyes undergoing pars plana vitrectomy. Posterior vitreous detachment status may be a prognostic factor for functional outcomes of surgery.

\section{Conflict of interest}

The authors declare no conflict of interest.

\section{References}

1 Smiddy WE, Flynn Jr HW. Vitrectomy in the management of diabetic retinopathy. Surv Ophthalmol 1999; 43: 491-507.

2 Eliott D, Lee MS, Abrams GW. Proliferative diabetic retinopathy: principles and techniques of surgical treatment. In: Ryan SJ (ed). Retina, 4th ed. CV Mosby Co: St Louis, MO, USA, 2006; 3rd Vol, p 2414.

3 Eliott D. Vitreoretinal attachments in proliferative diabetic retinopathy; effect on outcome. In: Vail Vitrectomy Meeting. Vail, CO, 2004.

4 Sebag J. Imaging vitreous. Eye 2002; 16: 429-438.

5 Morita H, Funata M, Tokoro T. A clinical study of the development of posterior vitreous detachment in high myopia. Retina 1995; 15: 117-124.

6 Kobayashi $\mathrm{H}$, Ogino N. Incidence of posterior vitreous detachment in healthy eyes with age, sex and refraction as variables. Jpn J Ophthalmol 1985; 39: 514-515.

7 Ye J, Zhou C, Du H, Jiang R, Min H. Study on the posterior vitreous detachment in patients with high myopia. Zhongguo Yi Хue Ke Xue Yuan Хие Baо 1999; 21: 472-477.

8 Guthoff R, Schrader W. Longterm results in surgical removal of subfoveal choroidal Neovascularization in age-related macular degeneration. Acta Ophthalmol Scand 2004; 82: 686-690.

9 Ikeda T, Sawa H, Koizumi K, Yasuhara T, Kinoshita S, Tano Y. Vitrectomy for proliferative diabetic retinopathy with asteoid hyalosis. Retina 1998; 18: 410-420.

10 Lewis H, Abrams GW, Blumenkrans MS, Campo RV. Vitrectomy for diabetic macular traction and edema associated with posterior hyaloidal traction. Ophthalmology 1992; 99: 753-759.

11 Williams JG, Trese MT, Williams GA, Hartzer MK. Autologous plasmin enzyme in the surgical management of diabetic retinopathy. Ophthalmology 2001; 108: 1902-1905; discussion 1905-1906.

12 Yorston D, Wickham L, Benson S, Bunce C, Sheard R, Charteris D. Predictive clinical features and outcomes of vitrectomy for proliferative diabetic retinopathy. $\mathrm{Br} J$ Ophthalmol 2008; 92: 365-368.

13 Yonemoto J, Ideta H, Sasaki K, Tanaka S, Hirose A, Oka C. The age of onset of posterior vitreous detachment. Graefe's Arch Clin Exp ophthalmol 1994; 232: 67-70.

14 Pierro L, Brancato R, Robino X, Lattanzio R, Jansen A, Calori G. Axial length in patients with diabetes. Retina 1999; 19: 401-404.

15 Dogru M, Inoue M, Nakamura M, Yamamoto M. Modifying factors related to asymmetric diabetic retinopathy. Eye 1998; 12: 929-933. 